\title{
TENSOR PRODUCTS AND RELATED QUESTIONS
}

BY

B. R. GELBAUM(1)

0 . Introduction. The subject of tensor products of linear spaces has received attention from authors with varying points of view $[2 ; 3 ; 4 ; 5 ; 8 ; 10]$. It is the purpose of this paper to offer some contributions to the systematic development of this area, especially as it is concerned with tensor products of Banach algebras. There are points at which digressions from the main theme appear to be in order, and the results of these digressions seem to have independent interest.

1. Tensor products. If $L_{1}$ and $L_{2}$ are two modules over a ring $R$ with identity ( $L_{1}$ a right $R$-module, $L_{2}$ a left $R$-module) then $L_{1} \otimes_{R} L_{2}$ is nothing more nor less than the quotient of the free abelian group $\left(L_{1}, L_{2}\right)$ based on $L_{1} \times L_{2}$ by the subgroup $N_{R}\left(L_{1}, L_{2}\right)$ generated by elements of the form $\left(l_{i}, l_{i}^{\prime}, l_{i}^{\prime \prime} \in L_{i}, r \in R\right)$ :

$$
\begin{aligned}
& \left(l_{1}^{\prime}+l_{1}^{\prime \prime}, l_{2}\right)-\left(l_{1}^{\prime}, l_{2}\right)-\left(l_{1}^{\prime \prime}, l_{2}\right), \\
& \left(l_{1}, l_{2}^{\prime}+l_{2}^{\prime \prime}\right)-\left(l_{1}, l_{2}^{\prime}\right)-\left(l_{1}, l_{2}^{\prime \prime}\right), \\
& \left(l_{1} r, l_{2}\right)-\left(l_{1}, r l_{2}\right) ;
\end{aligned}
$$

i.e., $L_{1} \otimes_{R} L_{2}=\left(L_{1}, L_{2}\right) / N_{R}\left(L_{1}, L_{2}\right)$ [1]. When $R$ is the real or complex number field and $L_{1}$ and $L_{2}$ are Banach spaces $E_{1}$ and $E_{2}$ or Banach algebras $A_{1}$ and $A_{2}$, the above definition takes no account of the essential aspects of these structures, that is of their norms. In $[2 ; 3 ; 5 \cdot 8 ; 10]$ are given various modifications of the above definition for Banach spaces and algebras. We summarize in

Definition 1. If $E_{1}$ and $E_{2}$ are Banach spaces, let, in general, $X^{Y}$ denote the set of all mappings from $Y$ to $X$, and let

$$
\begin{aligned}
F_{\gamma}\left(E_{1}, E_{2}\right)=\left\{f \mid f \in C^{E_{1} \times E_{2}}, f\left(0, x_{2}\right)\right. & =f\left(x_{1}, 0\right)=0, \\
& \left.\|f\| \equiv \sum_{\left(x_{1}, x_{2}\right)}\left|f\left(x_{1}, x_{2}\right)\right|\left\|x_{1}\right\|\left\|x_{2}\right\|<\infty\right\}
\end{aligned}
$$

where $C$ is the system of complex numbers, $x_{i} \in E_{i}, i=1,2$.

Regarded as an $L_{1}(\mu)$ space over $E_{1} \times E_{2}$, where $\mu\left(x_{1}, x_{2}\right)=\left\|x_{1}\right\|\left\|x_{2}\right\|$, $F_{\gamma}\left(E_{1}, E_{2}\right)$ is a Banach space.

If $E_{i}$ is a Banach algebra $A_{i}, i=1,2$, then $F_{\gamma}\left(A_{1}, A_{2}\right)$ may be made into

Presented to the Society, September 1, 1961; received by the editors June 6, 1961.

(1) The author is grateful to the National Science Foundation for its contribution to the support of the research reported herein. 
a Banach algebra by defining multiplication of two functions $f, g \in F_{\gamma}\left(A_{1}, A_{2}\right)$ as follows:

$$
\begin{aligned}
f * g\left(x_{1}, x_{2}\right) & =0, \quad \text { if } x_{1}=0 \text { or } x_{2}=0, \\
& =\sum_{a_{1} b_{1}=x_{1} ; a_{2} b_{2}=x_{2}} f\left(a_{1}, a_{2}\right) g\left(b_{1}, b_{2}\right), \text { otherwise. }
\end{aligned}
$$

As indicated in [3] this is a valid definition (the series above converges and $\left.f * g \in F_{\gamma}\left(A_{1}, A_{2}\right)\right)$.

To produce a normed structure akin to $L_{1} \otimes_{R} L_{2}$, we let $I$ be the closed linear subspace generated by functions $f$ satisfying one of the four conditions $(\alpha \in C)$ :

(i) $f\left(x_{1}, x_{2}^{\prime}\right)=f\left(x_{1}, x_{2}^{\prime \prime}\right)=-f\left(x_{1}, x_{2}^{\prime}+x_{2}^{\prime \prime}\right), f=0$ otherwise;

(ii) $f\left(x_{1}^{\prime}, x_{2}\right)=f\left(x_{1}^{\prime \prime}, x_{2}\right)=-f\left(x_{1}^{\prime}+x_{1}^{\prime \prime}, x_{2}\right), f=0$ otherwise;

(iii) $f\left(x_{1}, x_{2}\right)=-\alpha f\left(\alpha x_{1}, x_{2}\right), f=0$ otherwise;

(iv) $f\left(x_{1}, x_{2}\right)=-\alpha f\left(x_{1}, \alpha x_{2}\right), f=0$ otherwise;

and then define $E_{3} \equiv E_{1} \otimes_{\gamma} E_{2}$ as $F_{\gamma}\left(E_{1}, E_{2}\right) / I$. (Conditions (i)-(iv) correspond to the descriptions given earlier of the generators of $N_{R}\left(L_{1}, L_{2}\right)$.)

A simple computation, exploiting the fact that "finite" functions (i.e., functions vanishing at all but a finite number of points) are dense in both $F_{\gamma}\left(E_{1}, E_{2}\right)$ and $I$, reveals that when $E_{i}$ is a Banach algebra $A_{i}, i=1,2$, then $I$ is an ideal in the algebra $F_{\gamma}\left(A_{1}, A_{2}\right)$ whence $A_{3} \equiv A_{1} \otimes_{\gamma} A_{2}$ is a Banach algebra. The norm $\gamma$ on $E_{3}$ (quotient norm for the quotient space) satisfies the equation $\gamma\left(x_{1} \otimes x_{2}\right)=\left\|x_{1}\right\|\left\|x_{2}\right\|$.

As opposed to the usual constructions, the one described above produces $E_{1} \otimes_{\gamma} E_{2}$ as a (complete) quotient space of a complete space. (Customarily one norms equivalence classes of finite expressions $[5$, p. $28 ; 8]$ and then completes the resulting structure.)

There is another important tensor product for Banach spaces, viz., $E_{1} \otimes_{\lambda} E_{2}$ [8]. We shall define it by a direct construction:

Let

$$
\begin{array}{r}
F_{\lambda}\left(E_{1}, E_{2}\right)=\left\{f \mid f \in C^{E_{1} \times E_{2}}, \sum_{\left(x_{1}, x_{2}\right)} f\left(x_{1}, x_{2}\right) \lambda_{1}\left(x_{1}\right) \lambda_{2}\left(x_{2}\right)\right. \\
\text { convergent for all } \left.\left(\lambda_{1}, \lambda_{2}\right) \in E_{1}^{*} \times E_{2}^{*}\right\} .
\end{array}
$$

In the above

$$
\sum_{\left(x_{1}, x_{2}\right)} \cdots
$$

is taken to mean the unordered sum and convergence of

$$
\sum_{\left(x_{1}, x_{2}\right)} \cdots
$$


means convergence of the finite partial sums regarded as a net over the directed set of finite subsets of $E_{1} \times E_{2}$. A corollary to the Banach-Steinhaus theorem is the fact that for $f \in F_{\lambda}\left(E_{1}, E_{2}\right)$,

$$
\|f\| \equiv \sup _{\left\|\lambda_{1}\right\|,\left\|\lambda_{2}\right\| \leqq 1}\left|\sum_{\left(x_{1}, x_{2}\right)} f\left(x_{1}, x_{2}\right) \lambda_{1}\left(x_{1}\right) \lambda_{2}\left(x_{2}\right)\right|<\infty
$$

and with this seminorm $F_{\lambda}\left(E_{1}, E_{2}\right)$ is a (non- $\left.T_{2}\right)$ topological vector space. If $J$ is the closed linear hull of the generators used for $I$ before, then $F_{\lambda}\left(E_{1}, E_{2}\right) / J$ is precisely $E_{1} \otimes_{\lambda} E_{2}$, a Banach space. Again $\lambda\left(x_{1} \otimes x_{2}\right)=\left\|x_{1}\right\|\left\|x_{2}\right\|$. There seems to be no clear way of making $A_{1} \otimes_{\lambda} A_{2}\left(A_{1}, A_{2}\right.$ Banach algebras) into a Banach algebra. This last difficulty explains in part the favored role played by $\boldsymbol{\gamma}$.

Grothendieck $\left[5\right.$, p. 51] shows that $E_{1} \otimes_{\gamma} E_{2}$ may also be regarded as $F_{\gamma 0}\left(E_{1}, E_{2}\right) / I_{0}$, where

$$
\begin{array}{r}
F_{\gamma 0}\left(E_{1}, E_{2}\right)=\left\{f \mid f \in F_{\gamma}\left(E_{1}, E_{2}\right), f\left(x_{1}, x_{2}\right)=0 \text { if }\left\|x_{1}\right\| \text { or }\left\|x_{2}\right\|\right. \text { is greater than 1, } \\
\left.\qquad \sum_{\left(x_{1}, x_{2}\right)}\left|f\left(x_{1}, x_{2}\right)\right|<\infty\right\},
\end{array}
$$

and $I_{0}=F_{\gamma 0} \cap I$. We note that when $E_{1}$ and $E_{2}$ are Banach algebras $A_{1}$ and $A_{2}$, then $F_{\gamma 0}\left(A_{1}, A_{2}\right)$ is again an algebra and $I_{0}$ is a closed ideal in this algebra. We shall return to this point later.

Thus far we have offered no detailed proofs because these can be found in the literature $[3 ; 5$, loc. cit. $]$. There is however one remark that we give as a proposition together with its proof.

Proposition 1. Let $A_{1}$ and $A_{2}$ be Banach algebras. Then $F_{\gamma}\left(A_{1}, A_{2}\right)$ has an identity if and only if both $A_{1}$ and $A_{2}$ have identities.

Proof. The symbol $\delta_{\left(x_{1}, x_{2}\right)}$ will denote that member of $F_{\gamma}\left(E_{1}, E_{2}\right)$ equal to 1 at $\left(x_{1}, x_{2}\right)$ and 0 otherwise. It is clear that if $e_{i}$ is the identity of $A_{i}, i=1,2$, then $\delta_{\left(e_{1}, e_{2}\right)}$ is the identity in $F_{\gamma}\left(A_{1}, A_{2}\right)$.

On the other hand, let $f$ be the identity of $F_{\gamma}\left(A_{1}, A_{2}\right)$. For any $g \in F_{\gamma}\left(A_{1}, A_{2}\right), \bar{f} * g=(f * \bar{g})^{-}=\overline{\bar{g}}=g$, whence $f=\bar{f}$, i.e., $f$ is real. We show next that $f \geqq 0$.

First we note that neither $A_{1}$ nor $A_{2}$ has an annihilator other than 0 . For if $x_{1} A_{1}=0$, and $x_{1} \neq 0 \neq x_{2} \in A_{\dot{z}}$, then for

$$
\begin{aligned}
g & =\delta_{\left(x_{1}, x_{2}\right),} g * f\left(x_{1}, x_{2}\right)=1, \\
& =\sum_{x_{1} y_{1}=x_{1} ; x_{2} y_{2}-x_{2}} f\left(y_{1}, y_{2}\right) .
\end{aligned}
$$

But $x_{1} y_{1}=0 \neq x_{1}$, whence the right sum is empty and cannot equal 1 .

Now let $S=\left\{\left(c_{n}, d_{n}\right) \mid f\left(c_{n}, d_{n}\right) \neq 0\right\} . S$ is a countable set since 


$$
\sum_{\left(x_{1}, x_{2}\right)}\left|f\left(x_{1}, x_{2}\right)\right|\left\|x_{1}\right\|\left\|x_{2}\right\|<\infty .
$$

Let $\left\{t_{n}\right\}$ be an enumeration of all nonzero differences $c_{p}-c_{q},\left\{s_{n}\right\}$ an enumeration of all nonzero differences $d_{p}-d_{q}$. (If either $\left\{t_{n}\right\}$ or $\left\{s_{n}\right\}$ is empty an argument given below applies. This is a less interesting and less involved situation.)

Choose $k_{1} \in A_{1}$ such that $k_{1} t_{1} \neq 0$. If $k_{1} t_{2} \neq 0$, let $k_{2}=k_{1}$. If $k_{1} t_{2}=0$ let $\epsilon \in A_{1}$ be such that $\epsilon t_{2} \neq 0$. Then for $\|\epsilon\|$ small enough we can be certain that $k_{1}+\epsilon \equiv k_{2}$ satisfies:
(a) $\left\|k_{2} t_{1}\right\|>(1 / 2)\left\|k_{1} t_{1}\right\|$;
(b) $k_{2} t_{2} \neq 0$;
(c) $\left\|k_{2}-k_{1}\right\|<1 / 2$.

Having chosen $k_{1}, k_{2}, \cdots, k_{n}$ satisfying:

(a) $\left\|k_{n} t_{j}\right\|>(1 / 2)\left\|k_{j} t_{j}\right\|, j=1,2, \cdots, n-1$;

$\left(b_{1}\right) k_{n} t_{n} \neq 0$;

(c) $\left\|k_{j+1}-k_{j}\right\|<1 / 2^{i}, j=1,2, \cdots, n-1$;

choose $k_{n+1}$ to be $k_{n}$ if $k_{n} t_{n+1} \neq 0$. Otherwise let $\epsilon_{1} t_{n+1} \neq 0$ and then choose $\left\|\epsilon_{1}\right\|$ so small that $k_{n+1} \equiv k_{n}+\epsilon_{1}$ satisfies

(a2) $\left\|k_{n+1} t_{j}\right\|>(1 / 2)\left\|k_{j} t_{j}\right\|, j=1,2, \cdots, n$;

(b) $k_{n+1} t_{n+1} \neq 0$;

(c) $\left\|k_{n+1}-k_{n}\right\|<1 / 2^{n}$

Then $\lim _{n \rightarrow \infty} k_{n}=k$ exists, $\left\|k t_{j}\right\| \geqq(1 / 2)\left\|k_{j} t_{j}\right\|>0$, all $j$.

Similarly choose $l \in A_{2},\left\|l s_{j}\right\|>0$, all $j$, and let $g=\delta_{(k, l)}$. Since $f * g=g$, we see

$$
\sum_{c k=k ; d l=l} f(c, d)=1
$$

Our choice of $k$ and $l$ assures us that for only one pair $\left(c_{n_{0}}, d_{n_{0}}\right)$ in the range of summation is the term $f(c, d) \neq 0$, whence $f\left(c_{n_{0}}, d_{n_{0}}\right)=1$.

Assume $f\left(c_{n_{1}}, d_{n_{1}}\right)<0$ and let $h=M \delta_{(k, l)}+\delta_{\left(k c_{n_{1}}, l d_{n_{1}}\right)}$. Then

$$
\begin{aligned}
1=h\left(k c_{n_{1}}, l d_{n_{1}}\right) & =\sum_{x c=k c_{n_{1}} ; y d=l d_{n_{1}}} h(x, y) f(c, d) \\
& =M \sum_{k=k c_{n_{1}} ; l d=l d_{n_{1}}} f(c, d)+\sum_{k c_{n_{1}} c=k c_{n_{1}} ; l d_{n_{1}} d-l d_{n_{1}}} f(c, d) .
\end{aligned}
$$

Our choice of $k, l$ insures that the first sum consists of one term $\operatorname{Mf}\left(c_{n_{1}}, d_{n_{1}}\right)$. Hence the right member can be made arbitrarily large (negatively) since the second sum is finite and independent of $M$. This contradiction shows $f \geqq 0$ in this case.

In case one of the sets, say $\left\{t_{n}\right\}$, is empty, then all $c_{n}$ are equal, say to $c_{0}$. Thus for any $x_{1} \neq 0, x_{2} \neq 0, g=\delta_{\left(x_{1}, x_{2}\right)}$ we find

$$
\sum_{c_{0} y_{1}=x_{1} ; d_{n} y_{2}=x_{2}} f\left(c_{0}, d_{n}\right) g\left(y_{1}, y_{2}\right)=\sum_{c_{0} x_{1}=x_{1} ; d_{n} x_{2}=x_{2}} f\left(c_{0}, d_{n}\right)=1 .
$$


This means the sum is not empty and $c_{0} x_{1}=x_{1}$ for all $x_{1}$, and similarly $x_{1} c_{0}=x_{1}$ for all $x_{1}$, whence $c_{0}$ is an identity for $A_{1}$.

Again using $g=\delta_{\left(x_{1}, x_{2}\right)}$, we find from the equation $f * g=g$, that

$$
\sum_{c x_{1}=t_{1} ; d x_{2}=t_{2}} f(c, d)= \begin{cases}1, & \text { if } t_{1}=x_{1}, t_{2}=x_{2}, \\ 0, & \text { otherwise. }\end{cases}
$$

Since $f \geqq 0$, we see $f(c, d)=0$ if $c x_{1} \neq x_{1}$ or $d x_{2} \neq x_{2}$. Thus if $f(c, d)>0$, then $c x_{1}=x_{1}, d x_{2}=x_{2}$, any $x_{1}, x_{2}$. Similarly, from the equation $g * f=g$ we conclude $x_{1} c=x_{1} ; x_{2} d=x_{2}$ if $f(c, d)>0$. Thus if $f(c, d)>0, c$ and $d$ are (unique) identities for $A_{1}$ and $A_{2}$. In other words for only one pair $(c, d)$ is $f(c, d)>0, c$ is the identity of $A_{1}, d$ the identity of $A_{2}$ and $f(c, d)=\delta_{(c, d)}$.

A corollary to the proof is the fact that $F_{\gamma 0}\left(A_{1}, A_{2}\right)$ has an identity if and only if both $A_{1}$ and $A_{2}$ have identities.

Proposition 2.

(a) $\left(F_{\gamma}\left(E_{1}, E_{2}\right)\right)^{*}=\left\{\lambda\left|\lambda \in C^{E_{1} \times E_{2}},\right| \lambda\left(x_{1}, x_{2}\right) \mid \leqq K\left\|x_{1}\right\|\left\|x_{2}\right\|\right\}$.

(b) $\left(E_{1} \otimes_{\gamma} E_{2}\right)^{*}=\operatorname{Hom}_{b}\left(E_{1}, E_{2}^{*}\right)=\operatorname{Hom}_{b}\left(E_{2}, E_{1}^{*}\right)$.

(c) For $A_{1}, A_{2}$ commutative Banach algebras,

$$
\begin{aligned}
& \mathfrak{M}_{F_{\gamma}\left(A_{1}, A_{2}\right)}=\left\{\chi \mid \chi \in C^{A_{1} \times A_{2}}, \chi\left(a_{1}, a_{2}\right) \chi\left(b_{1}, b_{2}\right)=\chi\left(a_{1} b_{1}, a_{2} b_{2}\right),\right. \\
& \left.\left|\chi\left(a_{1}, a_{2}\right)\right| \leqq\left\|a_{1}\right\|\left\|a_{2}\right\|\right\} \text {. }
\end{aligned}
$$

(d) For $A_{1}, A_{2}$ commutative Banach algebras,

$$
\mathfrak{M}_{A_{1} \otimes \gamma A_{2}}=\left\{\chi \mid \chi \in \mathfrak{M}_{F_{\gamma}\left(A_{1}, A_{2}\right)}, \chi \text { bilinear }\right\}
$$

$[2 ; 3 ; 5$, p. $30 ; 6]\left(\operatorname{Hom}_{b}(\cdots)\right.$ and $\mathfrak{M} \cdots$ are explained in the proof).

Proof. We note first that the set $\left\{\delta_{\left(x_{1}, x_{2}\right)}\right\}$ is a basis for $F_{\gamma}\left(E_{1}, E_{2}\right)$ (and for $F_{\lambda}\left(E_{1}, E_{2}\right)$ ). Furthermore, for $f \in F_{\gamma}\left(E_{1}, E_{2}\right)$ (or $F_{\lambda}\left(E_{1}, E_{2}\right)$ ),

$$
f=\sum_{\left(x_{1}, x_{2}\right)} f\left(x_{1}, x_{2}\right) \delta_{\left(x_{1}, x_{2}\right)} \text {. }
$$

(a) If $\Lambda \in\left(F_{\gamma}\left(E_{1}, E_{2}\right)\right)^{*}$, let $\Lambda\left(\delta_{\left(x_{1}, x_{2}\right)}\right)=\lambda\left(x_{1}, x_{2}\right)$. Then $\left|\lambda\left(x_{1}, x_{2}\right)\right|$ $\leqq K\left\|\delta_{\left(x_{1}, x_{2}\right)}\right\|=K\left\|x_{1}\right\|\left\|x_{2}\right\|$ where $\|\Lambda\|=K$, and

$$
\Lambda(f)=\sum_{\left(x_{1}, x_{2}\right)} f\left(x_{1}, x_{2}\right) \lambda\left(x_{1}, x_{2}\right) .
$$

It is clear also that $K=\sup \left\{\left|\lambda\left(x_{1}, x_{2}\right)\right| /\left\|x_{1}\right\|\left\|x_{2}\right\| \mid x_{1}, x_{2} \neq 0\right\}$. Conversely, if $\left|\lambda\left(x_{1}, x_{2}\right)\right| \leqq K\left\|x_{1}\right\|\left\|x_{2}\right\|$, then $\Lambda$ defined by

$$
\Lambda(f)=\sum_{\left(x_{1}, x_{2}\right)} f\left(x_{1}, x_{2}\right) \lambda\left(x_{1}, x_{2}\right)
$$

is in $\left(F_{\gamma}\left(E_{1}, E_{2}\right)\right)^{*}$ and $\|\Lambda\|=\sup \left\{\left|\lambda\left(x_{1}, x_{2}\right)\right| /\left\|x_{1}\right\|\left\|x_{2}\right\| \mid x_{1}, x_{2} \neq 0\right\}$.

(b) Since $E_{1} \otimes_{\gamma} E_{2}=F_{\gamma}\left(E_{1}, E_{2}\right) / I, \quad\left(E_{1} \otimes_{\gamma} E_{2}\right)^{*}$ is precisely $I^{\perp} C$ $\left(F_{\gamma}\left(E_{1}, E_{2}\right)\right)^{*}$, i.e., the continuous linear functionals on $F_{\gamma}\left(E_{1}, E_{2}\right)$ that 
annihilate $I$. But $\Lambda \in I^{\perp}$ if and only if $\Lambda$ annihilates the generators of $I$. This implies that $\lambda\left(x_{1}, x_{2}\right)$ is a bilinear functional which means that $\lambda\left(x_{1}, x_{2}\right)$ for fixed $x_{2}$ may be regarded as an element of $E_{1}^{*}$ and that this association between $x_{2}$ and elements of $E_{1}^{*}$ is a bounded linear mapping. The set of such bounded linear mappings is denoted by $\operatorname{Hom}_{b}\left(E_{2}, E_{1}^{*}\right)$. It is clear that each element of $\operatorname{Hom}_{b}\left(E_{2}, E_{1}^{*}\right)$ corresponds to a $\Lambda \in I^{\perp}$ and that the correspondence is 1-1. By reversing the roles of $x_{1}$ and $x_{2}$ the equation $\left(E_{1} \otimes_{\gamma} E_{2}\right)^{*}=\operatorname{Hom}_{b}\left(E_{1}, E_{2}\right)^{*}$ is proved.

(c) $\mathfrak{M} \ldots$ denotes the space of regular maximal ideals of $\cdots$ The proofs of (c) and (d) are straight-forward extensions of the proofs of (a) and (b) when notice is taken of the multiplicative nature of the functionals involved [6].

2. Heredity. Before we pursue further developments of the ideas just presented, we shall, within the limited context thus far available, discuss the problem of heredity and a related problem.

Definition 1. Let $P$ be a property of Banach spaces in a certain class. (If the Banach space $E$ has property $P$ we write: $P(E)$.) $P$ is called hereditary under $\otimes_{\gamma}$ (or simply hereditary) if :

$$
P\left(E_{1}\right) \text { and } P\left(E_{2}\right) \Rightarrow P\left(E_{1} \otimes_{\gamma} E_{2}\right) .
$$

A simple example of an hereditary property $P$ is that of commutativity in the class of Banach algebras. We shall find below a number of hereditary properties $P$ together with a property $P$ for which the problem of heredity leads to extremely general and central problems in functional analysis.

THEOREM 1. The following properties are hereditary:

$\mathrm{P}_{1}$ : regularity (in the class of commutative Banach algebras);

$\mathrm{P}_{2}$ : density of $\mathfrak{F}^{-1}\left(C_{0}(\mathfrak{M})\right)$ (same); (see proof below for explanation of symbols);

$\mathrm{P}_{3}$ : presence of a basis (in the class of Banach spaces);

$\mathrm{P}_{4}$ : presence of a continuous involution (in the class of Banach algebras);

$\mathrm{P}_{5}$ : analyticity (in the class of commutative Banach algebras);

$\mathrm{P}_{6}$ : self-adjointness (in the class of commutative Banach algebras);

$\mathrm{P}_{7}$ : antisymmetry (in the class of commutative Banach algebras);

$\mathrm{P}_{8}$ : simplicity, i.e., absence of regular ideals (in the class of Banach algebras).

Proof. $\mathrm{P}_{1}$. Let $A_{1}$ and $A_{2}$ be regular (commutative) Banach algebras. From $[2 ; 3]$ we know that $\mathfrak{M}_{A_{1} \otimes \gamma A_{2}}$ and $\mathfrak{M}_{A_{1}} \times \mathfrak{M}_{A_{2}}$ are homeomorphic in a natural way when weak* topologies are used throughout. To prove $P_{1}\left(A_{1} \otimes_{\gamma} A_{2}\right)$ we choose a point $\left(M_{10}, M_{20}\right) \in \mathfrak{M}_{A_{1}} \times \mathfrak{M}_{A_{2}}$ and a neighborhood of the form $U_{1}\left(M_{10}\right) \times U_{2}\left(M_{20}\right)$. In $A_{i}$ we choose an $x_{i}$ such that $\hat{x}_{i}\left(M_{i 0}\right)=1$, $\hat{x}_{i}\left(M_{i}\right)=0, M_{i} \notin U_{i}\left(M_{i 0}\right), i=1,2$. Then clearly in $A_{1} \otimes_{\gamma} A_{2}$ the element $z$ represented by $x_{1} \otimes x_{2}$ has the property: $\hat{z}\left(M_{10}, M_{20}\right)=1, \hat{z}\left(M_{1}, M_{2}\right)=0$ if $\left(M_{1}, M_{2}\right) \notin U_{1}\left(M_{10}\right) \times U_{2}\left(M_{20}\right)$. 
$\mathrm{P}_{2}$. [10]. If $A$ is a commutative Banach algebra with maximal ideal space $\mathfrak{M}$, let $\mathfrak{F}: A \rightarrow C(\mathfrak{M})$ be the canonical map taking $x \in A$ into $\hat{x}(M)$ in $C(\mathfrak{M})$. Let $C_{0}(\mathfrak{M})$ be the set of functions of $C(\mathfrak{M})$ with compact support. Then $\mathfrak{F}^{-1}\left(C_{0}(\mathfrak{M})\right)$ is the set of elements $x$ in $A$ such that $\hat{x}(M)$ has compact support. The denseness of this set in $A$ is critical in the Wiener Tauberian theorem.

We now assume $\mathrm{P}_{2}$ obtains for $A_{1}$ and $A_{2}$ and begin with

LeMma 1. If $z$ in $A_{1} \otimes_{\gamma} A_{2}$ is represented by $x_{1} \otimes x_{2}$, then support $z=\left(\right.$ support $\left.\hat{x}_{1}\right) \times\left(\right.$ support $\left.\hat{x}_{2}\right)$.

Proof. Let $\left(M_{10}, M_{20}\right) \in\left(\right.$ support $\left.\hat{x}_{1}\right) \times\left(\right.$ support $\left.\hat{x}_{2}\right)$. If $\left(M_{10}, M_{20}\right) \notin$ support 2 , there are neighborhoods $U_{i}\left(M_{i 0}\right), i=1,2$ such that $\hat{z}\left(M_{1}, M_{2}\right)$ $\equiv \hat{x}_{1}\left(M_{1}\right) \hat{x}_{2}\left(M_{2}\right)=0$ for $\left(M_{1}, M_{2}\right) \in U_{1}\left(M_{10}\right) \times U_{2}\left(M_{20}\right)$. Hence either $\hat{x}_{1}\left(M_{1}\right) \equiv 0$ in $U_{1}\left(M_{10}\right)$ (which contradicts: $M_{10} \in$ support $\left.\hat{x}_{1}\right)$ or there is an $M_{11} \in U_{1}\left(M_{10}\right)$ such that $\hat{x}_{1}\left(M_{11}\right) \neq 0$. In the latter case $\hat{x}_{2}\left(M_{2}\right) \equiv 0$ in $U_{1}\left(M_{10}\right)$ and this second contradiction forces the conclusion: (support $\left.\hat{x}_{1}\right) \times\left(\right.$ support $\left.\hat{x}_{2}\right) \subset$ support $z$.

On the other hand, if $\left(M_{10}, M_{20}\right) \in$ support $z$ and, say, $M_{10} \notin$ support $\hat{x}_{1}$, then there is a neighborhood $U_{1}\left(M_{10}\right)$ such that $\hat{x}_{1}\left(M_{1}\right)=0$ in $U_{1}\left(M_{10}\right)$. Then for arbitrary $M_{2}, \hat{z}\left(M_{1}, M_{2}\right)=0,\left(M_{1} \in U_{1}\left(M_{10}\right)\right)$, and thus $\left(M_{10}, M_{20}\right) \notin$ support 2 .

We return to the proof proper. The essence of the idea is to show that elements in $A_{1} \otimes_{\gamma} A_{2}$ represented by finite sums of the form

$$
\sum_{\left(x_{1}, x_{2}\right)} f\left(x_{1}, x_{2}\right) \delta_{\left(x_{1}, x_{2}\right)}
$$

where $\hat{x}_{i}$ has compact support, $i=1,2$, whenever $f\left(x_{1}, x_{2}\right) \neq 0$, are dense in $A_{1} \otimes_{\gamma} A_{2}$. Thus let $z$ be arbitrary in $A_{1} \otimes_{\gamma} A_{2}$ and let it be represented by $f$. Then we may use the formal sum

$$
\sum_{n=1}^{\infty} a_{n}\left(x_{1 n} \otimes x_{2 n}\right),
$$

where $\left\{\left(x_{1 n}, x_{2 n}\right)\right\}_{n=1}^{\infty}$ is the set where $f$ is not zero and $a_{n}=f\left(x_{1 n}, x_{2 n}\right)$, to represent $z$.

For $\epsilon>0$, choose $u_{1 n}, u_{2 n}$ such that the supports of $a_{1 n}, a_{2 n}$ are compact and such that

$$
\left\|x_{1 n}-u_{1 n}\right\|<\epsilon / 6 \cdot 2^{n} K, \quad\left\|x_{2 n}-u_{2 n}\right\|<\epsilon / 6 \cdot 2^{n} K,
$$

where $K=\|f\|$. Set $x_{i n}=u_{i n}+v_{i n}, i=1,2, n=1,2, \cdots$. Then the formal sum

$$
\begin{aligned}
& \sum_{n=1}^{\infty} a_{n}\left(u_{1 n} \otimes u_{2 n}\right)+\sum_{n=1}^{\infty} a_{n}\left(u_{1 n} \otimes v_{2 n}\right) \\
+ & \sum_{n=1}^{\infty} a_{n}\left(v_{1 n} \otimes u_{2 n}\right)+\sum_{n=1}^{\infty} a_{n}\left(v_{1 n} \otimes v_{2 n}\right)
\end{aligned}
$$


also corresponds to a representative of $z$. Let the first group of terms of the formal sum represent $z_{\epsilon}$. Clearly $\gamma\left(z-z_{\epsilon}\right)<\epsilon / 2$. But for large enough $N$,

$$
\sum_{n=1}^{N} a_{n}\left(u_{1 n} \otimes u_{2 n}\right)
$$

represents a $z_{\epsilon N}$ with the property $\gamma\left(z-z_{\epsilon N}\right)<\epsilon$ and clearly $z_{\epsilon N}$ has compact support.

$\mathrm{P}_{3}$. This follows from results in [4].

$\mathrm{P}_{4}$. If * represents a continuous involution in each of $A_{1}$ and $A_{2}$, we can define an involution, again denoted by ${ }^{*}$, in $F_{\gamma}\left(A_{1}, A_{2}\right)$ by the formula:

$$
f^{*}\left(x_{1}, x_{2}\right)=\left(f\left(x_{1}^{*}, x_{2}^{*}\right)\right)^{-}
$$

Then

$$
\begin{aligned}
\left\|f^{*}\right\| & =\sum_{\left(x_{1}, x_{2}\right)}\left|\left(f\left(x_{1}^{*}, x_{2}^{*}\right)\right)-\right|\left\|x_{1}\right\|\left\|x_{2}\right\| \\
& \leqq K \sum_{\left(x_{1}, x_{2}\right)}\left|f\left(x_{1}^{*}, x_{2}^{*}\right)\right|\left\|x_{1}^{*}\right\|\left\|x_{2}^{*}\right\|
\end{aligned}
$$

where, if $\left\|x_{i}^{*}\right\| \leqq K_{i}\left\|x_{i}\right\|, i=1,2, K \geqq K_{1}, K_{2}$. Furthermore for $f \in I, f^{*} \in I$. Thus for $z \in A_{1} \otimes_{\gamma} A_{2}$ and $f$ representing $z$, the formula $z^{*}=\left(f^{*} / I\right)$ defines $z^{*}$ unambiguously. If $g_{n} \in I,\left\|f-g_{n}\right\| \rightarrow \gamma(z)$ as $n \rightarrow \infty$, then $\left\|f^{*}-g_{n}^{*}\right\| \leqq K\left\|f-g_{n}\right\|$, whence $\gamma\left(z^{*}\right) \leqq\left\|f^{*}-g_{n}^{*}\right\| \leqq K \gamma(z)$ and the involution is continuous.

$\mathrm{P}_{5}$. Assume $A_{1}$ and $A_{2}$ are analytic (commutative) Banach algebras. This means that if $x_{i} \in A_{i}$ and $\hat{x}_{i}\left(M_{i}\right)=0$ for all $M_{i}$ in an open set of $\mathfrak{M}_{\boldsymbol{A}_{i}}$, then $\hat{x}_{i} \equiv 0$. We must show that if $z \in A_{1} \otimes_{\gamma} A_{2}$ and $\hat{z}\left(M_{1}, M_{2}\right)$ is zero for all $\left(M_{1}, M_{2}\right)$ in an open set of $\mathfrak{M}_{A_{1} \otimes \gamma A_{2}}$, then $\hat{z} \equiv 0$. Thus if $\hat{z}=0$ on an open set, there are open sets $U_{i} \subset \mathfrak{M}_{A_{i}}, i=1,2$, such that $z=0$ on $U_{1} \times U_{2}$. Fix $M_{20} \in U_{2}$. Then $\mathcal{z}\left(M_{1}, M_{20}\right)$, regarded as a function on $\mathfrak{M}_{\mathbb{A}_{1}}$, actually arises from an element of $A_{1}$. Indeed if $z$ is represented by

$$
\sum_{n=1}^{\infty} a_{n}\left(x_{1 n} \otimes x_{2 n}\right)
$$

then the element

$$
x_{1}=\sum_{n=1}^{\infty} a_{n} \hat{x}_{2 n}\left(M_{20}\right) x_{1 n}
$$

of $A_{1}$ serves (the series converges absolutely). Hence $\hat{x}_{1} \equiv 0$ since $A_{1}$ is analytic. Similarly for $M_{10}$ fixed in $U_{1}, \hat{z}\left(M_{10}, M_{2}\right)$ regarded as a function on $\mathfrak{M}_{\Lambda_{2}}$ arises from the element

$$
x_{2}=\sum_{n=1}^{\infty} a_{n} \hat{x}_{1 n}\left(M_{10}\right) x_{2 n}
$$


of $A_{2}$ and thus $\hat{x}_{2} \equiv 0$. For arbitrary $M_{11} \in \mathfrak{M}_{A_{1}}$ we find then $\hat{z}\left(M_{11}, M_{2}\right)=0$ for $M_{2} \in U_{2}$, whence $\hat{z}\left(M_{11}, M_{2}\right)=0$ for arbitrary $M_{2}$ and the result follows.

$\mathrm{P}_{6}$. The proof of this resembles that for $\mathrm{P}_{4}$ and is omitted.

$\mathrm{P}_{7}$. A commutative Banach algebra $A$ is called antisymmetric if the existence of a pair $x, y$ in $A$ such that $\hat{x}=y^{-}$implies either that $x=y=0$ (modulo the radical) or that there is an identity $e$ in $A$ and that for some constant $\alpha, x=\alpha e, y=\bar{\alpha} e$. Now assume $z$ and $w$ in $A_{1} \otimes_{\gamma} A_{2}$ satisfy: $z=w^{-}$. As in the proof of $\mathrm{P}_{5}$, and in the notation of that proof, the elements $x_{1}$ and $x_{2}$ exist, and corresponding to $w$ there are elements $y_{1}$ and $y_{2}$. It follows that $\hat{x}_{1}=\hat{y}_{1}$, $\hat{x}_{2}=\hat{y}_{2}$, and a repetition of the argument in the proof of $\mathrm{P}_{5}$ then shows that either $z=w=0$ (modulo the radical) or that $A_{1} \otimes_{\gamma} A_{2}$ has an identity $e$ and that for some constant $\alpha, z=\alpha e, w=\bar{\alpha} e$.

$\mathrm{P}_{8}$. Assume $A_{1}$ and $A_{2}$ contain no regular ideals. Let $R$ be a regular ideal in $A_{1} \otimes_{\gamma} A_{2}$. Then there is a regular maximal ideal $M \supset R$ and $M$ engenders [3] regular maximal ideals $M_{1}$ and $M_{2}$ in $A_{1}$ and $A_{2}$. However, this contradicts the assumption of simplicity of $A_{1}$ and $A_{2}$.

TheOREM 2. Let $A_{1}$ and $A_{2}$ be commutative Banach algebras with Silov boundaries $\Gamma_{A_{1}}$ and $\Gamma_{A_{2}}$. When the maximal ideal space of $A_{1} \otimes_{\gamma} A_{2}$ is identified with $\mathfrak{M}_{A_{1}} \times \mathfrak{M}_{A_{2}}$, the Silov boundary of $A_{1} \otimes_{\gamma} A_{2}$ is mapped onto $\Gamma_{A_{1}} \times \Gamma_{A_{2}}$.

Proof. (a) $\Gamma_{A_{1}} \times \Gamma_{A_{2}} \subset \Gamma_{A_{1} \otimes \gamma A_{2}}$. Let $\left(M_{10}, M_{20}\right) \in \Gamma_{A_{1}} \times \Gamma_{A_{2}}$ and let $U\left(M_{10}, M_{20}\right)$ be a neighborhood of $\left(M_{10}, M_{20}\right)$. We can find neighborhoods $U_{1}\left(M_{10}\right)$ and $U_{2}\left(M_{20}\right)$ such that $U_{1}\left(M_{10}\right) \times U_{2}\left(M_{20}\right) \subset U\left(M_{10}, M_{20}\right)$. Then we can find $x_{i} \in A_{i}, i=1,2$, such that $\left|\hat{x}_{i}\right|$ reaches its maximum in $U_{i}\left(M_{i 0}\right)$ and is strictly smaller outside $U_{i}\left(M_{i 0}\right)$. Then $z$ represented by $x_{1} \otimes x_{2}$ is such that $|\hat{z}|$ reaches its maximum in $U_{1}\left(M_{10}\right) \times U_{2}\left(M_{20}\right)$ and is strictly smaller outside $U_{i}\left(M_{10}\right) \times U_{2}\left(M_{20}\right)$, a fortiori strictly smaller outside $U\left(M_{10}, M_{20}\right)$. Hence $\left(M_{10}, M_{20}\right) \in \Gamma_{A_{1} \otimes \gamma A_{2}}$.

(b) $\Gamma_{A_{1} \otimes \gamma A_{2}} \subset \Gamma_{A_{1}} \times \Gamma_{A_{2}}$. Let $z \in A_{1} \otimes_{\gamma} A_{2}$. Then for fixed $M_{2}, \hat{z}\left(M_{1}, M_{2}\right)$ $\in \hat{A}_{1}$ and thus

$$
\left|z\left(M_{1}, M_{2}\right)\right| \leqq \sup \left\{\left|\hat{z}\left(\gamma_{1}, M_{2}\right)\right| \gamma_{1} \in \Gamma_{A_{1}}\right\} .
$$

Let $\left|\hat{z}\left(\gamma_{10}, M_{2}\right)\right|=\sup \left|\hat{z}\left(M_{1}, M_{2}\right)\right|$. Then

$$
\begin{aligned}
\left|\hat{z}\left(\gamma_{10}, M_{2}\right)\right| & \leqq \sup \left\{\left|\hat{z}\left(\gamma_{10}, \gamma_{2}\right)\right| \mid \gamma_{2} \in \Gamma_{A_{2}}\right\} \\
& \leqq \sup \left\{\left|\hat{z}\left(\gamma_{1}, \gamma_{2}\right)\right| \mid\left(\gamma_{1}, \gamma_{2}\right) \in \Gamma_{A_{1}} \times \Gamma_{A_{2}}\right\}
\end{aligned}
$$

Hence for arbitrary $\left(M_{1}, M_{2}\right)$, we find

$$
\left|z\left(M_{1}, M_{2}\right)\right| \leqq \sup \left\{\left|z\left(\gamma_{1}, \gamma_{2}\right)\right| \mid\left(\gamma_{1}, \gamma_{2}\right) \in \Gamma_{A_{1}} \times \Gamma_{A_{2}}\right\},
$$

and this shows that $\Gamma_{A_{1} \otimes \gamma A_{2}} \subset \Gamma_{A_{1}} \times \Gamma_{A_{2}}$.

Conspicuously absent from the list of properties in Theorem 1 is the property of semisimplicity. Its omission is due to our inability to prove that semisimplicity is hereditary and not to the "fact" that semisimplicity is 
known not to be hereditary. Because of the ramifications of the problem, we shall treat it in a separate section.

3. Semisimplicity. By definition, a Banach algebra $A$ is semisimple if and only if its radical is $\{0\}$. The radical, for our purposes, is not the Jacobson radical but the intersection of all regular maximal two-sided ideals of $A$. Thus our basic question is: If $A_{1}$ and $A_{2}$ are semisimple, is $A_{1} \otimes_{\gamma} A_{2}$ semisimple? A partial solution to this problem has been found by Tomiyama [10] and was discovered independently by the present writer in the course of the research presented herein. (Note: If $A_{1} \otimes_{\gamma} A_{2}$ is semisimple, clearly so are $A_{1}$ and $A_{2 .}$ )

We saw earlier $(\S 1)$ that $A_{1} \otimes_{\gamma} A_{2}$ deserves special attention because it seems to be the tensor product that is also a nontrivial Banach algebra. When $A_{1}$ and $A_{2}$ are semisimple (which we shall assume hereafter in this section) the following theorem holds.

THEOREM 1. There is a tensor product $A_{1} \otimes_{\nu} A_{2}$ that is a semisimple Banach algebra. The norm $\nu$ is a cross-norm and the elements corresponding to representatives that are finite formal sums (not in $I$ )

$$
\sum_{n=1}^{K} a_{n}\left(x_{1 n} \otimes x_{2 n}\right),
$$

$K=1,2, \cdots$, are all nonzero and are dense in $A_{1} \otimes_{\nu} A_{2}$. The norm is of "general character" [8], $\lambda \leqq \nu \leqq \gamma$ and $\nu=\gamma$ if and only if $A_{1} \otimes_{\gamma} A_{2}$ is semisimple.

Proof. As we know from Proposition 2, $\S 1$ and its extension to the noncommutative case [3], $\mathfrak{M}_{A_{1} \otimes \gamma A_{2}}$ may be identified with a subset of $\mathfrak{M}_{F \gamma\left(A_{1}, A_{2}\right)}$, namely the maximal ideals annihilating $I$, i.e., hull $(I) \equiv h(I)[7]$. In the context of this identification, it is clear that

$$
I \subset k h(I)=\bigcap_{\mathfrak{M}_{\boldsymbol{A}_{1} \otimes_{\boldsymbol{\gamma}} \boldsymbol{A}_{2}}} M \equiv N
$$

The semisimplicity of $A_{1} \otimes_{\gamma} A_{2}$ is thus equivalent to the statement $I=N$. In any event $F_{\gamma}\left(A_{1}, A_{2}\right) / N$ is a Banach algebra whose norm $\nu$ is the usual quotient norm. We denote the result by $A_{1} \otimes_{\nu} A_{2}$.

Since the finite functions of $F_{\gamma}\left(A_{1}, A_{2}\right)$ are dense therein, it is clear that the elements of $A_{1} \otimes_{\nu} A_{2}$ with representatives that are finite formal sums are dense in $A_{1} \otimes_{\nu} A_{2}$. Furthermore, if $z$ has a finite representative not in $I$, we may choose it [8] to be

$$
\sum_{n=1}^{K} a_{n}\left(x_{1 n} \otimes x_{2 n}\right)
$$

where the sets $\left\{x_{1 n}\right\}_{1}^{K},\left\{x_{2 n}\right\}_{1}^{K}$ are both linearly independent. We show 


$$
\sum_{n=1}^{K} a_{n}\left(x_{1 n} \otimes x_{2 n}\right) \notin N .
$$

If it were in $N$, then we could read off the following conclusions when $A_{1}$ and $A_{2}$ are commutative:

$$
\begin{array}{rlrl}
\sum_{n=1}^{K} a_{n} \hat{x}_{1 n}\left(M_{1}\right) \hat{x}_{2 n}\left(M_{2}\right) & =0, & \text { all }\left(M_{1}, M_{2}\right) & \in \mathfrak{M}_{A_{1}} \times \mathfrak{M}_{A_{2}} \\
\sum_{n=1}^{K} a_{n} \hat{x}_{1 n}\left(M_{1}\right) x_{2 n} & =0, & \text { all } M_{1} \in \mathfrak{M}_{A_{1}}
\end{array}
$$

(since $A_{2}$ is semisimple);

$$
a_{n} \hat{x}_{1 n}\left(M_{1}\right)=0, \quad \quad \text { all } M_{1} \in \mathfrak{M}_{A_{1}}, n=1,2, \cdots, K
$$

(since $\left\{x_{2 n}\right\}_{1}^{K}$ is a linearly independent set). We may assume all $a_{n} \neq 0$ whence $x_{1 n}=0, n=1,2, \cdots, K$, since $A_{1}$ is semisimple and thus a contradiction results.

When $A_{1}$ and $A_{2}$ are not commutative, the reasoning runs this way:

$$
\sum_{n=1}^{K} a_{n}\left(\left(x_{1 n} / M_{1}\right) \otimes\left(x_{2 n} / M_{2}\right)\right)
$$

represents 0 in $\left(A_{1} / M_{1}\right) \otimes_{\gamma}\left(A_{2} / M_{2}\right)$ for all $\left(M_{1}, M_{2}\right)$. Hence

$$
\sum_{n=1}^{K} a_{n} \phi_{2}\left(x_{2 n} / M_{2}\right) x_{1 n} / M_{1}=0
$$

for all $\phi_{2} \in\left(A_{2} / M_{2}\right)^{*},\left(M_{2}\right.$ fixed) and all $M_{1}$. Hence

$$
\sum_{n=1}^{K} a_{n} \phi_{2}\left(x_{2 n} / M_{2}\right) x_{1 n}=0
$$

since $A_{1}$ is semisimple. Hence $x_{2 n} / M_{2}=0$, all $M_{2}$, whence $x_{2 n}=0$ since $A_{2}$ is semisimple, and a contradiction results.

To prove $\nu$ is a cross-norm we proceed as follows.

LEMMA 1. If

$$
\sum_{n=1}^{\infty} a_{n}\left(x_{1 n} \otimes x_{2 n}\right) \sim f \in N \subset F_{\gamma}\left(A_{1}, A_{2}\right)
$$

and if $\phi_{1} \in A_{1}^{*}$, then

$$
\sum_{n=1}^{\infty} a_{n} \phi_{1}\left(x_{1 n}\right) x_{2 n}=0 .
$$

Proof. The series clearly converges. If the sum is not zero, then, by virtue 
of the semisimplicity of $A_{2}$, there is an $M_{2} \in M_{\Lambda_{2}}$ such that

$$
\sum_{n=1}^{\infty} a_{n} \phi_{1}\left(x_{1 n}\right) x_{2 n} / M_{2} \neq 0 \text {. }
$$

Hence there is a $\Psi_{2} \in\left(A_{2} / M_{2}\right)^{*}$ such that

$$
\sum_{n=1}^{\infty} a_{n} \phi_{1}\left(x_{1 n}\right) \Psi_{2}\left(x_{2 n} / M_{2}\right) \neq 0 .
$$

Thus

$$
\sum_{n=1}^{\infty} a_{n} \Psi_{2}\left(x_{2 n} / M_{2}\right) x_{1 n} \neq 0
$$

and since $A_{1}$ is semisimple, there is an $M_{1} \in \mathbb{M}_{\Lambda_{1}}$ such that

$$
\sum_{n=1}^{\infty} a_{n} \Psi_{2}\left(x_{2 n} / M_{2}\right) x_{1 n} / M_{1} \neq 0 \text {. }
$$

For some $\Psi_{1} \in\left(A_{1} / M_{1}\right)$ then

$$
\left(\Psi_{1} \otimes \Psi_{2}\right) \sum_{n=1}^{\infty} a_{n}\left(\left(x_{1 n} / M_{1}\right) \otimes\left(x_{2 n} / M_{2}\right)\right) \neq 0 .
$$

This implies that if $w$ in $\left(A_{1} / M_{1}\right) \otimes_{\lambda}\left(A_{2} / M_{2}\right)$ is represented by

$$
\sum_{n=1}^{\infty} a_{n}\left(\left(x_{1 n} / M_{1}\right) \otimes\left(x_{2 n} / M_{2}\right)\right),
$$

then $\lambda(w)>0$ and hence $\gamma(w)>0$ when we regard $w$ as a member of $\left(A_{1} / M_{1}\right) \otimes_{\gamma}\left(A_{2} / M_{2}\right)$ [8]. Thus $f \notin N$ and the contradiction yields the result.

If now $z$ is represented by $x_{1} \otimes x_{2}$, we know $\nu(z) \leqq\left\|x_{1}\right\|\left\|x_{2}\right\|$. On the other hand if

$$
\sum_{n=1}^{\infty} a_{n}\left(x_{1 n} \otimes x_{2 n}\right)
$$

is in $N$ and if $\phi_{1} \in A_{1}^{*}$ satisfies $\left\|\phi_{1}\right\|=1, \phi_{1}\left(x_{1}\right)=\left\|x_{1}\right\|$, then setting

$$
x_{1} \otimes x_{2}+\sum_{n=1}^{\infty} a_{n}\left(x_{1 n} \otimes x_{2 n}\right)=\sum_{n=1}^{\infty} b_{n}\left(y_{1 n} \otimes y_{2 n}\right)
$$

we find, using Lemma 1,

$$
\left\|x_{1}\right\|\left\|x_{2}\right\|=\left\|\phi_{1}\left(x_{1}\right) x_{2}\right\|=\left\|\sum_{n=1}^{\infty} b_{n} \phi_{1}\left(y_{1 n}\right) y_{2 n}\right\| \leqq \sum_{n=1}^{\infty}\left|b_{n}\right|\left\|y_{1 n}\right\|\left\|y_{2 n}\right\| .
$$

Thus $\left\|x_{1}\right\|\left\|x_{2}\right\| \leqq \nu(z)$, i.e., $\left\|x_{1}\right\|\left\|x_{2}\right\|=\nu(z)$, and $\nu \leqq \gamma[10]$. 
Furthermore, if $z$ is represented by the finite formal sum

$$
\sum_{n=1}^{K} a_{n}\left(x_{1 n} \otimes x_{2 n}\right)
$$

and if $\phi_{i} \in A_{i}^{*},\left\|\phi_{i}\right\|=1, i=1,2$, we have

$$
\begin{aligned}
\left|\left(\phi_{1} \otimes \phi_{2}\right)(z)\right| & =\left|\left(\phi_{1} \otimes \phi_{2}\right)(z+w)\right|=\left|\left(\phi_{1} \otimes \phi_{2}\right) \sum_{n=1}^{\infty} b_{n}\left(y_{1 n} \otimes y_{2 n}\right)\right| \\
& \leqq \sum_{n=1}^{\infty}\left|b_{n}\right|\left\|y_{1 n}\right\|\left\|y_{2 n}\right\|
\end{aligned}
$$

where

$$
w \sim \sum_{n=1}^{\infty} c_{n}\left(w_{1 n} \otimes w_{2 n}\right) \in N
$$

and

$$
\sum_{n=1}^{K} a_{n}\left(x_{1 n} \otimes x_{2 n}\right)+\sum_{n=1}^{\infty} c_{n}\left(w_{1 n} \otimes w_{2 n}\right)=\sum_{n=1}^{\infty} b_{n}\left(y_{1 n} \otimes y_{2 n}\right) .
$$

Hence $\left|\left(\phi_{1} \otimes \phi_{2}\right)(z)\right| \leqq \nu(z)$, i.e., $\lambda(z) \leqq \nu(z)$.

If $A_{1} \otimes_{\gamma} A_{2}$ is semisimple, $I=N$ and $\nu=\gamma$. If $\nu=\gamma$, then for $f \in N$, $\nu(f / N)=\gamma(f / N)=0$, i.e., $f \in I$, and hence $N=I$.

REMARKs. 1. For arbitrary Banach spaces $E_{1}, E_{2}$ the above procedure can be modified to produce an $E_{1} \otimes_{\tilde{\nu}} E_{2}$. In this case we consider $\tilde{N}=\left\{f \mid f \in F_{\gamma}\left(E_{1}, E_{2}\right), \quad\left(\phi_{1} \otimes \phi_{2}\right)(f)=0\right.$, all $\left.\left(\phi_{1}, \phi_{2}\right) \in E_{1}^{*} \times E_{2}^{*}\right\}$, and define $E_{1} \otimes, E_{2}$ to be $F_{\gamma}\left(E_{1}, E_{2}\right) / \tilde{N}$. We shall return to this point later.

2. Reflection on Theorem 1 suggests the following procedure when $A_{1}$ and $A_{2}$ are commutative: For $z$ with a finite representative

$$
\sum_{n=1}^{K} a_{n}\left(x_{1 n} \otimes x_{2 n}\right)
$$

let

$$
\omega(z)=\sup \left\{\left|\sum_{n=1}^{K} a_{n} \hat{x}_{1 n}\left(M_{1}\right) \hat{x}_{2 n}\left(M_{2}\right)\right| \mid\left(M_{1}, M_{2}\right) \in \mathfrak{M}_{A_{1}} \times \mathfrak{M}_{A_{2}}\right\} .
$$

Then $\omega(z)$ is independent of the representative used for $z$. Furthermore, we may repeat an earlier argument to conclude that if $z$ has a representative

$$
\sum_{n=1}^{K} a_{n}\left(x_{1 n} \otimes x_{2 n}\right) \notin I
$$

then $\omega(z)>0$ (we assume $A_{1}$ and $A_{2}$ semisimple). $\omega$ is then a true norm on the 
algebraic tensor product $A_{1} \otimes A_{2}$ and the completion $A_{1} \otimes_{\omega} A_{2}$ can be shown to be a semisimple algebra with maximal ideal space $\mathfrak{M}_{\boldsymbol{A}_{1}} \times \mathfrak{M}_{\boldsymbol{A}_{2}}$. However, $\omega$ is not in general a cross-norm. This follows from:

$$
\omega(z)=\left\|x_{1}\right\|_{\infty}\left\|x_{2}\right\|_{\infty} \quad\left(z \sim\left(x_{1} \otimes x_{2}\right)\right),
$$

which is not in general $\left\|x_{1}\right\|\left\|x_{2}\right\|$, but $\leqq\left\|x_{1}\right\|\left\|x_{2}\right\|$.

The partial solution to the semisimplicity problem mentioned before is: $A_{1} \otimes_{\gamma} A_{2}$ is semisimple if either one of $A_{1}$ and $A_{2}$ satisfies the approximation condition [5, pp. 164-191; 10], viz., (for $A_{1}$ ) for $K_{1}$ compact in $A_{1}$ and $\epsilon>0$, there is a finite dimensional linear transformation $T_{1}$ defined on $A_{1}$ such that $\left\|x_{1}-T_{1} x_{1}\right\|<\epsilon$ for $x_{1} \in K_{1}$. In [5, pp. 164-191] there is an exhaustive discussion of equivalences and logical relations among the approximation condition and other phenomena. Particularly relevant is the equivalent condition of monomorphy: The natural mapping $\tau: A_{1} \otimes_{\gamma} E_{2} \rightarrow A_{1} \otimes_{\lambda} E_{2}$ is 1-1, for all Banach spaces $E_{2}$. (For each element $z$ of $A_{1} \otimes_{\gamma} E_{2}$ let $f \in F_{\gamma}\left(A_{1}, E_{2}\right)$ be a representative of $\boldsymbol{z}$. Then clearly $f \in F_{\lambda}\left(A_{1}, E_{2}\right)$ and represents an element $\boldsymbol{w}$ in $A_{1} \otimes_{\lambda} E_{2}$. It is clear that $w$ is independent of the choice of $f$, i.e., $w$ is uniquely determined by $z$, whence we write $w=\tau(z), \tau: A_{1} \otimes_{\gamma} E_{2} \rightarrow A_{1} \otimes_{\lambda} E_{2 .}$.) Our next result bears on this point. It was also observed by Robert Bonic in a letter to the writer.

ThEOREM 2. When $A_{1}$ and $A_{2}$ are commutative, $A_{1} \otimes_{\gamma} A_{2}$ is semisimple if and only if $\tau: A_{1} \otimes_{\gamma} A_{2} \rightarrow A_{1} \otimes_{\mathrm{\lambda}} A_{2}$ is 1-1.

Proof. Assume $A_{1} \otimes_{\gamma} A_{2}$ is semisimple and assume that $\tau(z)=0$. This means that for all $\left(\phi_{1}, \phi_{2}\right) \in A_{1}^{*} \times A_{2}^{*}$, and for any representative

$$
\sum_{n=1}^{\infty} a_{n}\left(x_{1 n} \otimes x_{2 n}\right)
$$

of $z$,

$$
\sum_{n=1}^{\infty} a_{n} \phi_{1}\left(x_{1 n}\right) \phi_{2}\left(x_{2 n}\right)=0
$$

In particular

$$
\sum_{n=1}^{\infty} a_{n} \hat{x}_{1 n}\left(M_{1}\right) \hat{x}_{2 n}\left(M_{2}\right)=0
$$

for all $M_{1}, M_{2}$, and hence $z=0$.

If $\tau$ is 1-1, and even if $A_{1}$ and $A_{2}$ are not commutative, we shall show that $A_{1} \otimes_{\gamma} A_{2}$ is semisimple. For if

$$
\sum_{n=1}^{\infty} a_{n}\left(\left(x_{1 n} / M_{1}\right) \otimes\left(x_{2 n} / M_{2}\right)\right)
$$


represents 0 for all $M_{1}, M_{2}$ and if

$$
\sum_{n=1}^{\infty} a_{n}\left(x_{1 n} \otimes x_{2 n}\right)
$$

does not represent 0 , there is a $\phi_{2} \in A_{2}^{*}$ such that

$$
\sum_{n=1}^{\infty} a_{n} \phi_{2}\left(x_{2 n}\right) x_{1 n} \neq 0 \text {. }
$$

Then there is an $M_{1}$ such that

$$
\sum_{n=1}^{\infty} a_{n} \phi_{2}\left(x_{2 n}\right)\left(x_{1 n} / M_{1}\right) \neq 0,
$$

and a $\Psi_{1} \in\left(A_{1} / M_{1}\right) *$ such that

$$
\sum_{n=1}^{\infty} a_{n} \phi_{2}\left(x_{2 n}\right) \Psi_{1}\left(x_{1 n} / M_{1}\right) \neq 0 .
$$

Hence

$$
\sum_{n=1}^{\infty} a_{n} x_{2 n} \Psi_{1}\left(x_{1 n} / M_{1}\right) \neq 0
$$

and for some $M_{2}$

$$
\sum_{n=1}^{\infty} a_{n}\left(x_{2 n} / M_{2}\right) \Psi_{1}\left(x_{1 n} / M_{1}\right) \neq 0 .
$$

Consequently there is a $\Psi_{2} \in\left(A_{2} / M_{2}\right)^{*}$ such that

$$
\sum_{n=1}^{\infty} a_{n} \Psi_{2}\left(x_{2 n} / M_{2}\right) \Psi_{1}\left(x_{1 n} / M_{1}\right) \neq 0
$$

Thus in $\left(A_{1} / M_{1}\right) \otimes_{\lambda}\left(A_{2} / M_{2}\right)$, the element 0 is represented by

$$
\sum_{n=1}^{\infty} a_{n}\left(\left(x_{1 n} / M_{1}\right) \times\left(x_{2 n} / M_{2}\right)\right)
$$

and yet $\lambda(0)>0$. The contradiction yields the result.

TheORem 3. When $A_{1}$ and $A_{2}$ are commutative semisimple Banach algebras, $A_{1} \otimes_{\gamma} A_{2}$ is both the completion of a tractable (i.e., not necessarily complete but semisimple) normed algebra and the quotient algebra of a semisimple Banach algebra.

Proof. Let $B$ be the image in $A_{1} \otimes_{\gamma} A_{2}$ of the finite functions of $F_{\gamma}\left(A_{1}, A_{2}\right)$. Then $B$ is dense in $A_{1} \otimes_{\gamma} A_{2}$ and, as we saw in the proof of Theorem $1, B$ is 
semisimple. (It is an easy matter to verify that $\mathfrak{M}_{B}$ is also $\mathfrak{M}_{\Lambda_{1}} \times \mathfrak{M}_{\Lambda_{2} \text {.) }}$ )

Let $S$ denote the semigroup consisting of the pairs

$$
\left\{\left(x_{1}, x_{2}\right) \mid x_{i} \in A_{i}, 0 \leqq\left\|x_{i}\right\| \leqq 1, i=1,2\right\}
$$

where $\left(x_{1}, x_{2}\right)\left(y_{1}, y_{2}\right)=\left(x_{1} y_{1}, x_{2} y_{2}\right)$. Then in the notation of [6] let

$$
l_{1}(S)=\left\{f\left|f \in C^{S},\|f\| \equiv \sum_{\left(x_{1}, x_{2}\right)}\right| f\left(x_{1}, x_{2}\right) \mid<\infty\right\} .
$$

In [6] it is shown that $l_{1}(S)$ is semisimple if and only if:

$$
\left(x_{1}, x_{2}\right)^{2}=\left(y_{1}, y_{2}\right)^{2}=\left(x_{1}, x_{2}\right)\left(y_{1}, y_{2}\right)
$$

implies $\left(x_{1}, x_{2}\right)=\left(y_{1}, y_{2}\right)$. In our case, when the displayed equations hold, we find $x_{1}^{2}=y_{1}^{2}=x_{1} y_{1}$ and thus $\left(x_{1}-y_{1}\right)^{2}=0$. Similarly $\left(x_{2}-y_{2}\right)^{2}=0$. Since $A_{1}$ and $A_{2}$ are semisimple, $x_{1}=y_{1}, x_{2}=y_{2}$ and we conclude $l_{1}(S)$ is semisimple.

We define a surjection $\sigma: l_{1}(S) \rightarrow F_{\gamma 0}\left(A_{1}, A_{2}\right)$ as follows: For $f \in l_{1}(S)$, let $\sigma f=g$ where

$$
\begin{aligned}
& g\left(0, x_{2}\right)=g\left(x_{1}, 0\right)=0, \\
& g\left(x_{1}, x_{2}\right)=f\left(x_{1}, x_{2}\right),
\end{aligned}
$$$$
x_{1}, x_{2} \neq 0 \text {. }
$$

We see at once that $\sigma$ is a norm-decreasing homomorphic surjection. Since there is a homomorphism of $F_{\gamma 0}\left(A_{1}, A_{2}\right)$ onto $A_{1} \otimes_{\gamma} A_{2}$ the result follows.

REMARKs. 1. A simple rewording of definitions shows that if $B_{1}$ is a $\mathrm{Ba}-$ nach algebra, $I$ a closed ideal in $B_{1}$, and if $B_{2}=B_{1} / I$, then the inverse image of the radical of $B_{2}$ is $k h(I)$. Thus the semisimplicity of $B_{2}$ is equivalent to the "spectral synthesis" of $I: I=k h(I)$ [7]. Since spectral synthesis is known to fail [P. Malliavin, Inst. des Hautes Etudes Scientifiques, Publications Math., No. 2, 61-68] in many Banach algebras, it follows that there are semisimple Banach algebras $A$ such that for some ideal $I \subset A, A / I$ is not semisimple.

2. The closure of a tractable algebra need not be semisimple.

For example, if

$$
A=\left\{\left\{a_{n}\right\}\left|a_{n} \in C,\left\|\left\{a_{n}\right\}\right\| \equiv \sum_{n=1}^{\infty}\right| a_{n} \mid 2^{-n^{2}}<\infty\right\},
$$

then relative to convolution as multiplication $A$ is a commutative Banach algebra. The element $x=\{0,1,0,0, \cdots\}$ in $A$ is such that $\left\|x^{n}\right\|=2^{-n^{2}}$ and thus $\left\|x^{n}\right\| 1 / n=2^{-n} \rightarrow 0$. Hence $A$ is not semisimple. On the other hand, the elements of $A$ with finite support form a dense subalgebra isomorphic to the algebra of polynomials in one variable. This algebra is tractable and thus we have a nonsemisimple algebra $A$ that is the closure of a tractable algebra. Dr. John Lindberg in a paper not yet published has given another example of this phenomenon in the area of algebraic extensions of Banach algebras. 
(Note: If $x=\left\{a_{n}\right\} \neq 0$ in $A$, and if $a_{n_{1}}$ is the first nonzero component then

$$
\left\|x^{2}\right\| \geqq\left|a_{n_{1}}^{2}\right| 2^{-n_{1}^{2}}>0 .
$$

Hence $A$ is free of nonzero elements $x$ such that $x^{2}=0$. In analogy with the proof of the second statement of Theorem 3, $A=l\left(S_{1}\right) / N$ where $S_{1}=\{x \mid 0 \leqq\|x\| \leqq 1\}$ and $N$ is a closed ideal in $l_{1}(S)$. Thus $A$ is also a nonsemisimple quotient algebra of a semisimple algebra $l_{1}(S)$.)

Theorem 4. When $A_{1}$ and $A_{2}$ are commutative and semisimple, $A_{1} \otimes_{\gamma} A_{2}$ has an identity if and only if both $A_{1}$ and $A_{2}$ have identities.

Proof. It is clear from direct inspection that $A_{1} \otimes_{\gamma} A_{2}$ has an identity if both $A_{1}$ and $A_{2}$ have identities.

On the other hand, if

$$
\sum_{n=1}^{\infty} a_{n}\left(x_{1 n} \otimes x_{2 n}\right)
$$

represents an identity in $A_{1} \otimes_{\gamma} A_{2}$, then

$$
\sum_{n=1}^{\infty} a_{n} \hat{x}_{1 n}\left(M_{1}\right) \hat{x}_{2 n}\left(M_{2}\right) \equiv 1
$$

Hence

$$
x_{2} \equiv \sum_{n=1}^{\infty} a_{n} \hat{x}_{1 n}\left(M_{1}\right) x_{2 n}
$$

for any $M_{1}$ is an identity modulo any $M_{2}$. Thus for any $y_{2} \in A_{2}, x_{2} y_{2}-y_{2}$ $\in \cap_{\mathfrak{P}_{A_{2}}} M_{2}=\{0\}$. Hence $x_{2}$ is an identity for $A_{2}$ and is independent of $M_{1}$. Similarly

$$
\sum_{n=1}^{\infty} a_{n} \hat{x}_{2 n}\left(M_{2}\right) x_{1 n}
$$

is an identity for $A_{1}$ and is independent of $M_{2}$.

Apropos of the discussion of the existence of an identity in $A_{1} \otimes_{\gamma} A_{2}$, the following may be observed: If $A_{1}$ and $A_{2}$ are commutative Banach algebras with identities, then $A_{1} \otimes_{\gamma} A_{2}$ is the direct sum of two ideals if and only if one of $A_{1}$ and $A_{2}$ is the direct sum of two ideals. This follows from Silov's theorem [9], from the equation $\mathfrak{M}_{\Lambda_{1} \otimes \gamma A_{2}}=\mathfrak{M}_{A_{1}} \times \mathfrak{M}_{A_{2}}$ and from the fact that $\mathfrak{M}_{A_{1}} \times \mathfrak{M}_{A_{2}}$ is connected if and only if both $\mathfrak{M}_{A_{1}}$ and $\mathfrak{M}_{\boldsymbol{A}_{2}}$ are connected.

We shall continue a discussion of the central problem of semisimplicity. However, at this point, the algebraic aspects of $A_{1}$ and $A_{2}$ will no longer intervene and we shall be concentrating on the purely "Banach-space" sides of the problem. 
4. Monomorphy. In the preceding section we defined the condition of monomorphy for a Banach $E_{1}[5, \mathrm{pp} .36,164-191]$.

THEOREM 1. Each of the following statements implies that $E_{1}$ satisfies the condition of monomorphy:

(a) There is a constant $K$ with the following property: If $C\left(S_{1}^{*}\right)$ denotes the space of continuous functions on the unit ball $S_{1}^{*}$ of $E_{1}^{*}$, and if $E_{1}$ is regarded as isometrically embedded in $C\left(S_{1}^{*}\right)$, then for any finite set $\left\{x_{i}\right\}_{i=1}^{m} \subset E_{1}$ and functions $\left\{f_{j}\right\}_{j=1}^{n} \subset C\left(S_{1}^{*}\right)$ for which

$$
x_{i}=\sum_{j=1}^{n} a_{i j} f_{j}, \quad i=1,2, \cdots, m
$$

( $a_{i j}$ constants), there exist elements $\left\{y_{j}\right\}_{j=1}^{n} \subset E_{1}$ such that $\left\|y_{j}\right\| \leqq K\left\|f_{j}\right\|$ and

$$
x_{i}=\sum_{j=1}^{n} a_{i j} y_{j}, \quad i=1,2, \cdots, m .
$$

(b) There is a bounded projection $P$ of $F\left(E_{1}\right)$ onto $N_{1}$ where $F\left(E_{1}\right)$ $=\left\{f\left|f \in C^{E_{1}},\|f\| \equiv \sum_{x}\right| f(x) \mid\|x\|<\infty\right\}, N_{1}=\left\{f \mid f \in F\left(E_{1}\right), \sum_{x} f(x) x=0\right\}$.

(c) There is no matrix $C=\left\{c_{i j}\right\}_{i, j=1}^{\infty}$ satisfying:

(i) $C^{2}=0$;

(ii) $\left|c_{i j}\right| \leqq a_{i} a_{j}, a_{i} \geqq 0, \quad \sum_{i=1}^{\infty} a_{i}^{2}<\infty$;

(iii) $\sum_{i=1}^{\infty} c_{i i}=1$.

((c) actually implies the condition of monomorphy for all Banach spaces.)

Proof. Assume (a) is true and let $E_{2}$ be an arbitrary Banach space for which $\tau: E_{1} \otimes_{\gamma} E_{2} \rightarrow E_{1} \otimes_{\lambda} E_{2}$ is not 1-1. This means there is an $f \in F_{\gamma}\left(E_{1}, E_{2}\right)$,

$$
f \sim \sum_{n=1}^{\infty} a_{n}\left(x_{1 n} \otimes x_{2 n}\right)
$$

such that for any pair $\left(\phi_{1}, \phi_{2}\right) \in E_{1}^{*} \times E_{2}^{*}$,

$$
\sum_{n=1}^{\infty} a_{n} \phi_{1}\left(x_{1 n}\right) \phi_{2}\left(x_{2 n}\right)=0
$$

and yet $f \notin I$.

Now regard $E_{1}$ as isometrically embedded in $C\left(S_{1}^{*}\right) \equiv F_{1}$. It is then clear that for $\left(\Psi_{1}, \phi_{2}\right) \in F_{1}^{*} \times E_{2}^{*}$,

$$
\sum_{n=1}^{\infty} a_{n} \Psi_{1}\left(x_{1 n}\right) \phi_{2}\left(x_{2 n}\right)=0 .
$$

This means that $f$, regarded as a member of $F_{\gamma}\left(F_{1}, E_{2}\right)$ via the obvious embedding, must be in $J$, the closed linear subspace of $F_{\gamma}\left(F_{1}, E_{2}\right)$ used to define $F_{1} \otimes_{\gamma} E_{2},\left(F_{\gamma}\left(F_{1}, E_{2}\right) / J=F_{1} \otimes_{\gamma} E_{2}\right)$, because $F_{1}[5$, pp. 185-191] fulfills the condition of monomorphy. This in turn means, that for $\epsilon>0$, there are gener- 
ators $g_{1}, g_{2}, \cdots, g_{n(\epsilon)}$ of $J$ and coefficients $b_{1}(\epsilon), \cdots, b_{n(\epsilon)}^{(\epsilon)}$ such that

$$
\left\|f-\sum_{i=1}^{n(\epsilon)} b_{i}(\epsilon) g_{i}\right\|<\epsilon / K \text {. }
$$

We may write

$$
f-\sum_{i=1}^{n(\epsilon)} b_{i}(\epsilon) g_{i} \sim \sum_{n=1}^{\infty} c_{n}\left(y_{1 n} \otimes y_{2 n}\right)+\sum_{m=1}^{n(\epsilon)} d_{m}\left(z_{1 m} \otimes z_{2 m}\right)
$$

where $y_{1 n} \in E_{1}, z_{1 m} \in F_{1} \backslash E_{1}$. Clearly for finitely many $y_{1 n_{i}}$ there are relations of the form

$$
y_{1 n_{i}}=\sum_{j=1}^{n(e)} a_{i j} z_{1 j}, \quad i=1,2, \cdots, p .
$$

If, in accordance with the condition, we replace each $z_{1 j}$ by some $w_{1 j} \in E_{1}$ such that

$$
y_{1 n_{i}}=\sum_{j=1}^{n(\epsilon)} a_{i j} w_{1 j}, \quad\left\|w_{1 j}\right\| \leqq K\left\|z_{1 j}\right\|
$$

then the $g_{1}, \cdots, g_{n(\epsilon)}$ will be replaced by $h_{1}, \cdots, h_{n(\epsilon)}$ in $I$ and

$$
\begin{aligned}
\left\|f-\sum_{i=1}^{n(\epsilon)} b_{i}(\epsilon) h_{i}\right\| & =\sum_{n=1}^{\infty}\left|c_{n}\right|\left\|y_{1 n}\right\|\left\|y_{2 n}\right\|+\sum_{m=1}^{n(\epsilon)}\left|d_{m}\right|\left\|w_{1 m}\right\|\left\|z_{2 m}\right\| \\
& \leqq K\left\|f-\sum_{i=1}^{n(\epsilon)} b_{i}(\epsilon) g_{i}\right\|<\epsilon .
\end{aligned}
$$

(We may and do assume $K \geqq 1$.) Thus $f$ is in fact in $I$, and a contradiction yields the result.

Assume (b) is true. Since $F\left(E_{1}\right)$ is essentially $L_{1}(\mu)$ where $\mu$ is discrete measure on $E_{1}\left(\mu\left(x_{1}\right)=\left\|x_{1}\right\|\right), F\left(E_{1}\right)$ satisfies the condition of monomorphy [5, loc. cit.]. Clearly the mapping $T: F\left(E_{1}\right) \rightarrow E_{1}$ defined by the formula $T(f)=\sum_{x} f(x) x$ is a continuous surjection and $T^{-1}(0)=N_{1}$. If $P\left(F\left(E_{1}\right)\right)=N_{1}$, then $(I-P)\left(F\left(E_{1}\right)\right)$ is isomorphic to $E_{1}$ ( $I$ is the identity operator). Since $(I-P)$ is a bounded projection we shall prove our result by establishing the following

LEMMA 1. If $E$ is a Banach space satisfying the condition of approximation, and if $P$ is a bounded projection of $E$ onto a closed linear subspace $N$, then $N$, qua Banach space, satisfies the condition of approximation.

Proof. Let $K$ be a compact set in $N$ and let $\epsilon>0$. Then $K$ is compact in $E$ and there is a finite dimensional linear transformation $S$ such that for $x \in K$, $\|x-S x\|<\epsilon /\|P\|$. Let $S^{\prime}=P S$. Then $S^{\prime}$ is finite dimensional, $S^{\prime}(N) \subset N$, and for $x \in K,\left\|x-S^{\prime} x\right\|=\|P x-P S x\| \leqq\|P\|\|x-S x\|<\epsilon$. 
Next assume (c) is true and let $E_{1}, E_{2}$ be arbitrary Banach spaces. If $\tau$ is not $1-1$ for the pair in question there is an

$$
f \sim \sum_{n=1}^{\infty} b_{n}\left(x_{1 n} \otimes x_{2 n}\right)
$$

in $F_{\gamma}\left(E_{1}, E_{2}\right)$ such that $f \notin I$ and

$$
\sum_{n=1}^{\infty} b_{n} \phi_{1}\left(x_{1 n}\right) \phi_{2}\left(x_{2 n}\right)=0
$$

all $\left(\phi_{1}, \phi_{2}\right) \in E_{1}^{*} \times E_{2}^{*}$. Since $f \notin I$, from the Hahn-Banach theorem we see that there is (Proposition 2, §1) a linear mapping $\lambda: E_{2} \rightarrow E_{1}^{*}$ such that

$$
\sum_{n=1}^{\infty} b_{m}\left\langle x_{1 n}, \lambda\left(x_{2 n}\right)\right\rangle=1
$$

We may represent $f$ by

$$
\sum_{n=1}^{\infty}\left(b_{1} x_{1 n} \otimes x_{2 n}\right) \equiv \sum_{n=1}^{\infty}\left(y_{1 n} \otimes x_{2 n}\right)
$$

and then again by

$$
\sum_{n=1}^{\infty}\left(\alpha_{n} y_{1 n} \otimes\left(1 / \alpha_{n}\right) x_{2 n}\right), \quad \quad \alpha_{n} \neq 0
$$

If

$$
\alpha_{n}=\left(\left\|x_{2 n}\right\| /\left\|y_{1 n}\right\|\right)^{1 / 2}
$$

then

$$
\left\|\alpha_{n} y_{1 n}\right\|=\left(\left\|x_{2 n}\right\|\left\|y_{1 n}\right\|\right)^{1 / 2}
$$

and

$$
\left\|\left(1 / \alpha_{n}\right) x_{2 n}\right\|=\left(\left\|x_{2 n}\right\|\left\|y_{1 n}\right\|\right)^{1 / 2} \text {. }
$$

Setting $u_{1 n}=\alpha_{n} y_{1 n}, v_{1 n}=\left(1 / \alpha_{n}\right) x_{2 n}$, we see $\left\|u_{1 n}\right\|=\left\|v_{1 n}\right\|$ and

$$
\sum_{n=1}^{\infty}\left\|u_{1 n}\right\|^{2}=\sum_{n=1}^{\infty}\left\|v_{1 n}\right\|^{2}<\infty .
$$

Clearly we may assume

$$
\sum_{n=1}^{\infty}\left\|u_{1 n}\right\|^{2}=1
$$

Thus we may and do assume $b_{n}=1$, 


$$
\sum_{n=1}^{\infty}\left\|x_{1 n}\right\|^{2}=\sum_{n=1}^{\infty}\left\|x_{2 n}\right\|^{2}=1, \quad\left\|x_{1 n}\right\|=\left\|x_{2 n}\right\| .
$$

Set $c_{m n}=\left\langle x_{1 m}, \lambda\left(x_{2 n}\right)\right\rangle$. Then $\left|c_{m n}\right| \leqq\left\|x_{1 m}\right\|\|\lambda\|\left\|x_{2 n}\right\|$. Thus if

$$
a_{n}=(\|\lambda\|)^{1 / 2}\left\|x_{1 n}\right\|
$$

we see

$$
\sum_{n=1}^{\infty} a_{n}^{2}<\infty, \quad\left|c_{m n}\right|<a_{m} a_{n}
$$

We see too that

$$
\sum_{n=1}^{\infty} \phi_{1}\left(x_{1 n}\right) x_{2 n}=0,
$$

all $\phi_{1} \in E_{1}^{*}$. Since $\lambda$ is a linear mapping we find

$$
\sum_{n=1}^{\infty} \phi_{1}\left(x_{1 n}\right)\left\langle x_{1 m}, \lambda\left(x_{2 n}\right)\right\rangle=0, \quad \text { all } \phi_{1} \in E_{1}^{*},
$$

and thus

$$
\sum_{n=1}^{\infty} c_{m n} x_{1 n}=0, \quad m=1,2, \cdots
$$

Finally

$$
0=\left\langle\sum_{n=1}^{\infty} c_{m n} x_{1 n}, \lambda\left(x_{2 k}\right)\right\rangle=\sum_{n=1}^{\infty} c_{m n} c_{n k} .
$$

Thus the conditions (i)-(iii) are fulfilled and we have a contradiction.

REMARKs. 1. For a given Banach space $E_{1},(\mathrm{a})$ is implied by the existence of a bounded projection $P: C\left(S_{1}^{*}\right) \rightarrow E_{1}$. Such a projection exists when $E_{1}=C(X)$ for some topological space $X$. (Embed $X$ in $S_{1}^{*}$ and let $P$ be the restriction mapping.) Of course, when $E_{1}=C(X)$, it is hardly necessary to find $P$ in order to establish the condition of monomorphy.

2. In $\left[5\right.$, p. $\left.171,\left(f^{\prime \prime}\right)\right]$ a condition analogous to (c) is discussed. We note that such a $C$ can not be in trace class since such a $C$ is unitarily equivalent to a matrix $D=\left(d_{m n}\right)$ with diagonal elements 0 . If $C$ were in the trace class,

$$
0=\sum_{n=1}^{\infty} d_{n n}=\sum_{n=1}^{\infty} c_{n n}=1
$$

$C$ is dominated, entry by entry, by the matrix $A=\left(a_{m} a_{n}\right)$ and $A$ is in the trace class $\left(A=B B^{t}\right.$ where $B$ is the Hilbert-Schmidt matrix $\left(b_{i j}\right) ;\left(b_{i 1}=a_{i}, b_{i j}=0\right.$, $j>1))$. Furthermore, it is possible to have: $\left|c_{m n}\right| \leqq t_{m n},\left(t_{m n}\right)=T$ in the trace class, $C$ not in the trace class. This is implicit in [4]. There it is shown that 
if $\left\{x_{i}\right\}$ is an orthonormal basis for $l_{2}$, then $\left\{x_{i} \otimes x_{j}\right\}$ is not an unconditional basis for $l_{2} \otimes_{\gamma} l_{2}$ which is known to be the set of trace class operators in $l_{2}$. It is also shown that $\left\{x_{i} \otimes x_{j}\right\}$ is an unconditional basis for $l_{2} \otimes_{\gamma} l_{2}$ if and only if for every sequence $\left\{a_{i}\right\} \in l_{2}$, the matrix $\left(\epsilon_{i j} a_{i} a_{j}\right)$ is in the trace class for all matrices $\left(\epsilon_{i j}\right),\left|\epsilon_{i j}\right| \leqq 1$.

5. $E_{1} \otimes_{i} E_{2}$. Let $\alpha$ be a cross-norm and consider the following situation: For each $z$ in $E_{1} \otimes_{\alpha} E_{2}$ such that $z$ has a finite representative, say

$$
\sum_{n=1}^{m} a_{n}\left(x_{1 n} \otimes x_{2 n}\right)
$$

let $w$ be the element of $E_{1} \otimes_{\lambda} E_{2}$ with the same representative. As in an earlier discussion, we note that $w$ is independent of the choice of representative, i.e., $w=\tau_{0}(z)$. If $\tau_{0}$ (obviously a linear transformation) is bounded, it can be extended to a mapping $\tau_{\alpha}: E_{1} \otimes_{\alpha} E_{2} \rightarrow E_{1} \otimes_{\lambda} E_{2}$. When we write $\tau_{\alpha}$ in the theorem below, we assume that $\tau_{0}$ is bounded.

THEOREM 1. If $\tau_{\alpha}$ is $1-1$, then $\alpha \leqq \tilde{\nu} ; \tilde{\nu}=\gamma$ if and only if $\tau: E_{1} \otimes_{\gamma} E_{2}$ $\rightarrow E_{1} \otimes_{\lambda} E_{2}$ is 1-1.

Proof. If $f$ is a finite function in $F_{\gamma}\left(E_{1}, E_{2}\right)$, let $T_{0} f$ be the element of $E_{1} \otimes_{\alpha} E_{2}$ represented by $f$. Since $\alpha$ is a cross-norm, $\alpha\left(T_{0} f\right) \leqq\|f\|$, i.e., $T_{0}$ is a bounded linear transformation from the (incomplete) normed space of finite functions in $F_{\gamma}\left(E_{1}, E_{2}\right)$ to $E_{1} \otimes_{\alpha} E_{2}$, and $\left\|T_{0}\right\| \leqq 1$. (Since $\alpha$ is a cross-norm, $\left\|T_{0}\right\|=1$.) Let $T$ be the extension of $T_{0}$ to all $F_{\gamma}\left(E_{1}, E_{2}\right)$. Again $\alpha(T f) \leqq\|f\|$. Let $\hat{N}=T^{-1}(0)$, and, as in Remark 1 following Theorem 1 , 33 , let $\tilde{N}=\left\{f \mid f \in F_{\gamma}\left(E_{1}, E_{2}\right),\left(\phi_{1} \otimes \phi_{2}\right)(f)=0\right.$, all $\left.\left(\phi_{1}, \phi_{2}\right) \in E_{1}^{*} \times E_{2}^{*}\right\}$.

We show $\tilde{N} \subset \hat{N}$. For if $f \in \tilde{N}$, then

$$
\begin{aligned}
\left\|\tau_{\alpha}(T f)\right\| & =\lim _{n \rightarrow \infty}\left\|\tau_{\alpha}\left(T f_{n}\right)\right\| \\
& =\lim _{n \rightarrow \infty} \sup \left\{\left|\sum_{m=1}^{n} a_{m} \phi_{1}\left(x_{1 m}\right) \phi_{2}\left(x_{2 m}\right)\right| \mid\left(\phi_{1}, \phi_{2}\right) \in S_{1}^{*} \times S_{2}^{*}\right\}
\end{aligned}
$$

where

$$
f \sim \sum_{m=1}^{\infty} a_{m}\left(x_{1 m} \otimes x_{2 m}\right), \quad f_{n} \sim \sum_{m=1}^{n} a_{m}\left(x_{1 m} \otimes x_{2 m}\right) .
$$

But for $\left(\phi_{1}, \phi_{2}\right) \in S_{1}^{*} \times S_{2}^{*}$, since $f \in \tilde{N}$

$$
\begin{aligned}
\left|\sum_{m=1}^{n} a_{m} \phi_{1}\left(x_{1 m}\right) \phi_{2}\left(x_{2 m}\right)\right| & \leqq\left|\sum_{m=1}^{\infty} a_{m} \phi_{1}\left(x_{1 m}\right) \phi_{2}\left(x_{2 m}\right)\right|+\left|\sum_{m=n+1}^{\infty} a_{m} \phi_{1}\left(x_{1 m}\right) \phi_{2}\left(x_{2 m}\right)\right| \\
& \leqq 0+\sum_{m=n+1}^{\infty}\left|a_{m}\right|\left\|x_{1 m}\right\|\left\|x_{2 m}\right\| .
\end{aligned}
$$

Since the last sum approaches 0 , independently of $\left(\phi_{1}, \phi_{2}\right)$ as $n \rightarrow \infty$ we see 
$\left\|\tau_{\alpha}(T f)\right\|=0$, i.e., $\tau_{\alpha}(T f)=0$, whence $T f=0$ since $\tau_{\alpha}$ is assumed to be 1-1.

We now resort to the following elementary result, which we prove for the sake of completeness.

LEMMA 1. If $S$ is a bounded linear transformation $S: F_{1} \rightarrow F_{2}$ where $F_{i}$ are Banach spaces, $i=1,2$, and if $K_{1}=S^{-1}(0)$, then for any $x_{1} \in F_{1},\left\|S\left(x_{1}\right)\right\|$ $\leqq\|S\| \rho\left(x_{1}, K_{1}\right)$, where $\rho\left(x_{1}, K_{1}\right)$ denotes the distance of $x_{1}$ from $K_{1}$.

Proof. For any $k_{1} \in K_{1}, S\left(x_{1}+k_{1}\right)=S\left(x_{1}\right)$, whence

$$
\left\|S\left(x_{1}\right)\right\|=\left\|S\left(x_{1}+k_{1}\right)\right\| \leqq\|S\|\left\|x_{1}+k_{1}\right\| .
$$

Hence $\left\|S\left(x_{1}\right)\right\| \leqq\|S\| \rho\left(x_{1}, K_{1}\right)$.

In our situation we find $\alpha(T f) \leqq \rho(f, \hat{N}) \leqq \rho(f, \hat{N})$. This means $\alpha(T f)$ $\leqq \tilde{\nu}(T f)$, and this is the desired result.

Otherwise stated: $\tilde{\nu}$ is the greatest of cross-norms $\alpha$ for which $\tau_{\alpha}$ is definable and 1-1. Clearly $\tilde{\nu}=\boldsymbol{\gamma}$ is the necessary and sufficient condition that $\tau$ be $1-1$.

TheOREM 2. Let $A_{1}$ and $A_{2}$ be semisimple commutative Banach algebras. Then $\tau_{\nu}: A_{1} \otimes_{\nu} A_{2} \rightarrow A_{1} \otimes_{\lambda} A_{2}$ is 1-1 and $\nu \leqq \tilde{\nu}$. Finally, there is an injection $\eta: A_{1} \otimes_{\nu} A_{2} \rightarrow A_{1} \otimes_{\omega} A_{2}$, and $A_{1} \otimes_{\omega} A_{2}$ is semisimple.

Proof. For $z \in A_{1} \otimes_{\nu} A_{2}$ such that $z$ has a finite representative $f$ in $F_{\gamma}\left(A_{1}, A_{2}\right)$, let $w$ be the element of $A_{1} \otimes_{\lambda} A_{2}$ with the same representative. Then $w$ is uniquely defined by $z, w=\tau_{0}(z)$ and we know $\lambda(w) \leqq \nu(z)$. Thus $\tau_{0}$ may be extended to $\tau_{\nu}$ defined on all $A_{1} \otimes_{\nu} A_{2}$.

Now assume $z$ in $A_{1} \otimes_{\nu} A_{2}$ has representative

$$
f \sim \sum_{n=1}^{\infty} a_{n}\left(x_{1 n} \otimes x_{2 n}\right)
$$

in $F_{\gamma}\left(A_{1}, A_{2}\right)$, and let $\tau_{\nu}(z)=0$. This means that

$$
\left|\sum_{n=1}^{N} a_{n} \phi_{1}\left(x_{1 n}\right) \phi_{2}\left(x_{2 n}\right)\right| \rightarrow 0
$$

uniformly for $\left(\phi_{1}, \phi_{2}\right)$ in $S_{1}^{*} \times S_{2}^{*}$, and this implies $f \in N, z=0$. From Theorem 1 we see $\nu \leqq \tilde{\nu}$.

Similarly we define $\eta_{0}$ on those members $z$ of $A_{1} \otimes_{\nu} A_{2}$ with finite representatives in $F_{\gamma}\left(A_{1}, A_{2}\right): \eta_{0}(z)$ is that element $w$ of $A_{1} \otimes_{\omega} A_{2}$ with the same representative as that of $z$ ( $w$ is independent of the choice of representative). $\eta_{0}$ is bounded and serves to define an $\eta: A_{1} \otimes, A_{2} \rightarrow A_{1} \otimes_{\omega} A_{2}$. If $\eta(z)=0$, let

$$
f \sim \sum_{n=1}^{\infty} a_{n}\left(x_{1 n} \otimes x_{2 n}\right)
$$

represent $z$ and let 


$$
\sum_{n=1}^{N} a_{n}\left(x_{1 n} \otimes x_{2 n}\right)
$$

represent $z_{N}$. Then $z_{N} \rightarrow z$ and $z_{N} \rightarrow 0$ uniformly on $\mathfrak{M}_{A_{1} \otimes \gamma A_{2}}$. Thus $\nu(z)=0$, i.e., $z=0, \eta$ is an injection. The proof that $A_{1} \otimes_{\omega} A_{2}$ is semisimple proceeds similarly.

Problem. If $\nu=\tilde{\nu}$ is $A_{1} \otimes_{\gamma} A_{2}$ semisimple?

\section{BIBLIOGRAPHY}

1. N. Bourbaki, Algebre, Hermann, Paris, 1948.

2. B. Gelbaum, Tensor products of Banach algebras, Canad. J. Math. 11 (1959), 297-310.

3. - Note on the tensor product of Banach algebras, Proc. Amer. Math. Soc. 12 (1961), 750-757.

4. B. Gelbaum and J. Gil de Lamadrid, Bases and tensor products of Banach spaces, Pacific J. Math. 11 (1961), 1281-1286.

5. A. Grothendieck, Products tensoriels topologiques et espaces nucleaires, Mem. Amer. Math. Soc. No. 16 (1955).

6. E. Hewitt and H. S. Zuckerman, The $l_{1}$-algebra of a commutative semigroup, Trans. Amer. Math. Soc. 83 (1956), 70-97.

7. L. Loomis, Introduction to abstract harmonic analysis, Van Nostrand, New York, 1953.

8. R. Schatten, $A$ theory of cross-spaces, Annals of Mathematics Studies No. 26, Princeton Univ. Press, Princeton, N. J., 1950.

9. G. E. Silov, On decomposition of a commutative normed ring in a direct sum of ideals, Mat. Sb. 32 (1953), 353-364 (Russian).

10. J. Tomiyama, Tensor products of commutative Banach algebras, Tôhoku Math. J. 12 (1960), 147-154.

INSTITUTE FOR ADVANCEd STUdy,

Princeton, New Jersey

UNIVERSITY OF MINNESOTA,

MinNEAPOLIS, MinNESOTA 